\title{
Atherosclerosis in Rheumatoid Arthritis: What to Look for in Studies Using Carotid Ultrasound
}

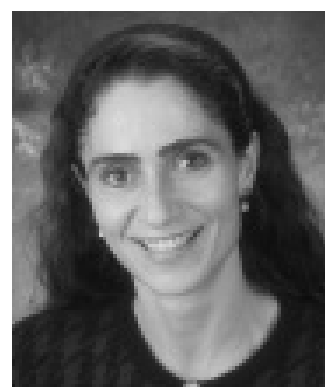

Studies of myocardial infarction and other cardiovascular (CV) events in patients with rheumatoid arthritis (RA) have consistently found that their incidence is at least twice as high as in controls without $\mathrm{RA}^{1-6}$. However, there is still uncertainty about the cause and pathophysiology of CV disease in RA. One reason is that, in contrast to $\mathrm{CV}$ event findings, previous studies on the extent of atherosclerosis in RA have not consistently found it is increased in RA.

In this issue of The Journal, Kobayashi and colleagues used carotid ultrasound to assess atherosclerosis in RA patients and controls ${ }^{7}$. In this commentary, I will first discuss the key technical aspects necessary for a high quality study using ultrasound; I will then briefly discuss published studies, focusing on those conducted in North American patient samples, and will end with a discussion of the article in this issue.

\section{Technical Requirements of Carotid Ultrasound}

High resolution carotid ultrasound has been used extensively to study atherosclerosis in the general population ${ }^{8}$. It provides a noninvasive, readily available method to image the major arteries of the neck. Its validity as a measure of atherosclerosis is supported by 3 lines of evidence: (1) the correlation between ultrasound and histological measurements of arterial intima-media thickness $\left(\right.$ IMT) ${ }^{9}$; (2) the correlation between histological measurements of carotid and coronary wall $\mathrm{IMT}^{10}$; and (3) the association between CV risk factors and ultrasound measurements of carotid IMT, and the ability of the latter to predict CV events in the general population $^{8,11}$. A number of technical requirements must be present for the ultrasound results to be valid, as itemized below:

Sample size. Ultrasound uses ultra-high frequency sound waves that bounce, radar-like, off the body's tissues to produce an image. Not surprisingly, this approach is limited by measurement error in its ability to visualize small structures. The arterial wall is near the resolution limits of ultrasound ${ }^{12}$, a limitation that can be overcome by studying a sufficiently large number of individuals, to reduce statistical error. Most studies of atherosclerosis in the general population have included samples in the several thousands. Comparable numbers are difficult to achieve studying RA, but this does not diminish the importance of studying a sufficient sample size. A small sample size detracts from a study's credibility, even if it reports statistically significant findings. Investigators should plan accordingly and document the power of their studies.

Adjustment for $C V$ risk factors. Even with large sample sizes, ultrasound is unable to separate the intimal layer, where atherosclerotic plaques occur, from the other layers of the arterial wall ${ }^{12}$. Instead, ultrasound measures the thickness of the intimal and media layers combined, the carotid IMT. This is important because the medial layer is sensitive to increased pressure in the lumen, responding by hypertrophy. Thus, a thickened IMT in the presence of hypertension may not reflect the presence of atheromatous plaque. Comparisons between RA patients and controls should account for $\mathrm{CV}$ risk factors through specification of enrollment criteria, matching, or statistical adjustment.

Standardized scanning protocol. The ultrasonographer should follow a rigorous, standardized scanning protocol that is identical for all RA patients and controls. This includes using the same equipment and programmed settings for both groups. This insures that images are of the same quality and from the same arterial segments, angles, and views in all subjects. Research studies should establish a process to certify technicians' proficiency with the scanning protocol.

Standardized reading protocol. Measurements should be obtained from a recorded video image, stilled at the end of diastole, to ensure that measurements are not influenced by cardiac cycle fluctuations. The IMT measurements are performed using software to manually trace the contours of the lumen-intima and media-adventitia interfaces (Figure 1).

See April issue Increased prevalence of carotid artery atherosclerosis in RA is artery-specific, page 730

Personal non-commercial use only. The Journal of Rheumatology Copyright @ 2010 . All rights reserved. 


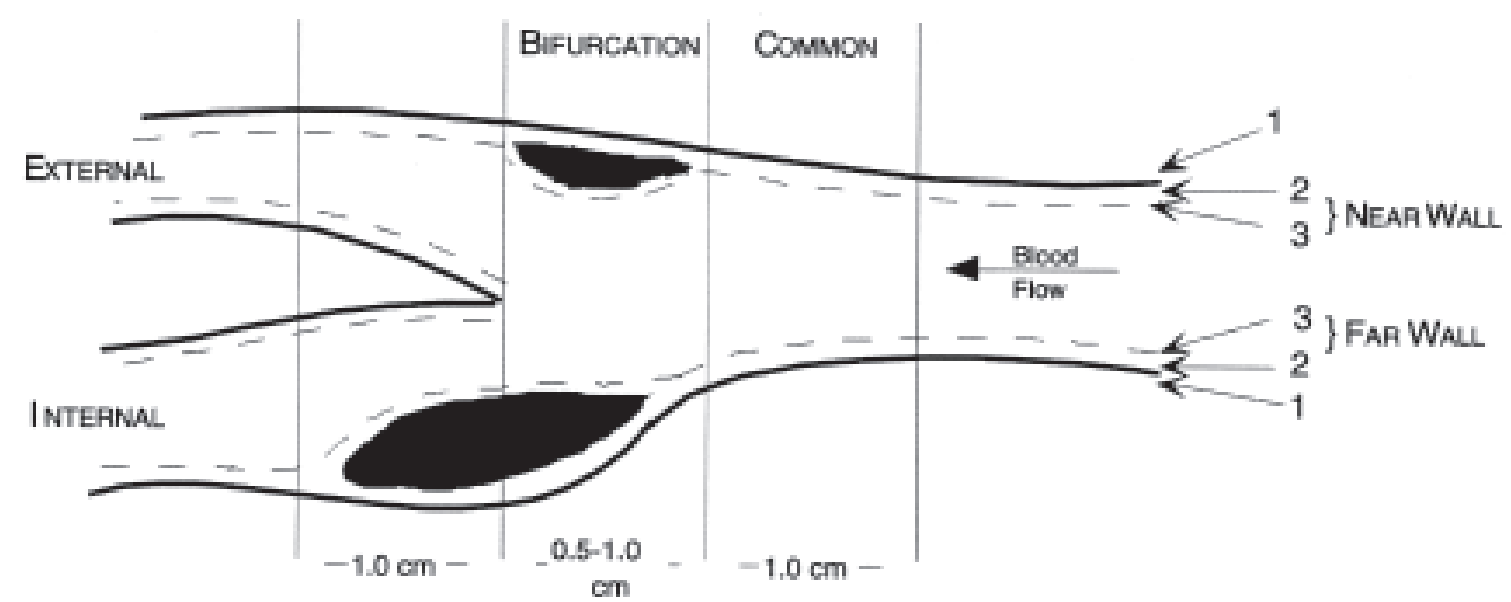

Figure 1. The carotid artery at the bifurcation. The interfaces traced to calculate the carotid intima-media thickness (IMT) are identified: 1. adventitia-periadventitia; 2. media-adventitia; 3 . lumen-intima. IMT is provided by the distance between lines 2 and 3 in each wall. In the study by Kobayashi, et $a l^{7}$, RA was associated with increased IMT in the internal, but not the common, carotid arteries.

The software then calculates the distance between the tracings in millimeters. Measurements are obtained from the common carotid artery, proximal to the bifurcation, and from the bulb and internal carotid. Turbulent flow at the bifurcation promotes deposition of atheromata in the bulb. The common carotid usually remains free of plaque, and displays lower IMT values than does the common carotid artery. When present, plaque is usually found in the carotid bulb and internal carotid artery. Most studies include plaque in their measurements of IMT. However, some studies have excluded plaque from IMT estimates ${ }^{13}$, resulting in lower IMT values. Because atheromata may display focal areas of increased thickness, multiple projections are imaged. For the internal carotid artery, two oblique and one lateral view of both the near and far wall of the artery are obtained. In the common carotid, deposits tend to be smoother, and thus only one projection each of the near and far walls is obtained. The multiplicity of values is usually reduced by averaging all the values, to produce a single IMT value per person. Alternatively, the internal and common carotid IMT values can be averaged separately, as done by Kobayashi, et $a l^{7}$. The inter- and intrarater reliability of the US measurements should be documented for the personnel, equipment, and subjects of the study in question. It is not sufficient to describe the reliability of the technique in the published literature.

Blinding. Reading a scan for IMT and plaque is operator-dependent, and susceptible to bias. It is important for the individual who reads the images not to know whether the image belongs to an RA patient or a control. RA and other rheumatic diseases are often evident on sight. Thus, to ensure blinded reading: (a) the reader should not be the same person who performs the scan; (b) RA patients and controls should be scanned concurrently using the same equipment. If this is not the case, changes in US equipment and technology may make it obvious to the reader what group the scan stems from; and (c) scans from cases and controls should be shuffled and read concurrently.

\section{Earlier North American Studies}

Investigators from throughout the world have used carotid ultrasound to estimate the extent of atherosclerosis in RA. Most of this literature is cited by Kobayashi and colleagues ${ }^{7}$. The results have been varied, and to date it has not been possible to infer conclusively whether atherosclerosis is increased in RA. Many of the studies have included modest sample sizes, detracting from conclusions derived from them. Here, I will limit my comments to studies of North American patients ${ }^{13-15}$, as these have included larger study samples, and may be more relevant to Kobayashi and colleagues' study, as the CV risk factor pattern and ethnic mix in North America may differ from those of other regions. In the first of these studies, my colleagues and I studied 204 RA patients and 102 controls matched for age and sex, all nonsmokers ${ }^{14}$. Their ages ranged from 40 to 83 years, and $89 \%$ were women. Cases and controls were studied concurrently, and scans were read blindly at a remote location, and adjusted for $\mathrm{CV}$ risk factors. Plaque was present in $30 \%$ of arteries imaged from RA patients, and in 25\% of those from controls. On a per-person basis, $44 \%$ of the RA patients and $38 \%$ of the controls had plaque, a difference that was not statistically significant with our sample size. We reported the composite common and internal carotid IMT as a single value per subject, which we obtained by averaging 16 IMT measurements of the internal and external carotid arteries. We found a difference of $0.055 \mathrm{~mm}$ between RA patients and controls, also not significant ${ }^{14}$.

Three years later, Roman and colleagues reported the Personal non-commercial use only. The Journal of Rheumatology Copyright @ 2010 . All rights reserved. 
results of a study of 96 women and 2 men with RA with ages ranging from 20 to 80 years ${ }^{13}$, whom they compared to an equal number of age, sex, and ethnicity-matched controls. The controls had participated in earlier longitudinal studies of hypertension, and were not studied concurrently to the RA patients ${ }^{13}$. The authors reported plaque was significantly more frequent in RA than controls, $44 \%$ versus $15 \%$. They did not include areas of plaque in their IMT measurements, thus IMT values were lower in the RA group ${ }^{13}$.

Most recently, Schott, et al reported their findings in 93 women with RA aged 42 to 60 years, matched by age, race, and menopausal status to 93 women without $\mathrm{RA}^{15}$. They found plaque in $21 \%$ of the RA patients and $15 \%$ of the controls, not a significant difference. For the IMT, they focused only on the common carotid artery, finding that it was not significantly different between the 2 groups. However, these authors reported a novel finding: arterial diameters were significantly greater in the RA patients, suggesting premature vascular aging ${ }^{15}$.

The above 3 studies reported variable associations between the carotid US findings and inflammation markers or $\mathrm{CV}$ risk factors used as covariates ${ }^{13-15}$. It should be noted, however, that these case-control comparisons are not ideally suited to study of association between atherosclerosis and covariates. Matching of cases and controls in these studies required specification of inclusion and exclusion criteria that may have truncated the covariate distribution, which may attenuate correlations. Thus, it cannot be concluded from them that $\mathrm{CV}$ risk factors are not associated with atherosclerosis in RA. In our study of 631 RA patients recruited consecutively, and without specifying $\mathrm{CV}$ risk factors as exclusion criteria, we reported that both $\mathrm{CV}$ risk factors and inflammation markers were significantly associated with carotid IMT and plaque ${ }^{16}$.

\section{This Issue of The Journal}

The studies described above show a mixed picture, making it difficult to infer conclusively whether or not carotid atherosclerosis is increased in RA. This brings us to the article by Kobayashi, et $a l^{7}$. Methodologically and technically, this study meets the highest standards of quality. The authors began with the end in mind, by specifying enrollment criteria identical to those of the Multi-Ethnic Study of Atherosclerosis (MESA), a large, population-based study of atherosclerosis, with the ultimate intent of comparing their RA sample to MESA. They studied 198 RA patients and 198 matched controls; ages ranged from 45 to 84 years, and the proportion of men was greater than in any of the other North American studies, $40 \%$. The technical aspects of the US were identical to those of MESA. Patients and controls were studied concurrently, by the same personnel, using the same equipment. This adds weight to the claim that readings were blinded. The authors imaged both the internal carotid artery bulb and the external carotid artery, and stratified their com- parisons between patients and controls according to artery. This enabled their key observation, that IMT was significantly greater among RA patients only in the bulb, but not in the common carotid. The frequency of plaque was also significantly greater in the RA group, $21.5 \%$ versus $12.1 \%$. Of note, the bulb IMT difference was significant only among men. This may explain some differences with previous studies, which included a greater proportion of women. Also notable, the bulb IMT and plaque difference between RA and controls was present only among subjects under 65 years of age. These results are consistent with our finding that RA manifestations contributed more to carotid atherosclerosis in young RA patients ${ }^{16}$, and with Roman and colleagues' finding of a more pronounced difference between RA patients and controls among younger subjects ${ }^{13}$.

Evaluating these 4 North American carotid US RA studies $^{7,13-15}$, it is evident that, in contrast to the consistently increased incidence of clinical CV events reported by different studies $^{1-6}$, a difference in carotid atherosclerosis between RA patients and controls has not been documented as consistently. Why may this be the case? One potential explanation is that RA patients may be only mildly predisposed to atherosclerosis, but more prone to develop unstable plaque, one that is more prone to ulcerate and thrombose. In support of this hypothesis, Aubry, et al reported an autopsy study in which RA patients displayed less histologic evidence of atherosclerosis, but more inflammation and instability compared to controls ${ }^{17}$.

What to do with this information? Kobayashi, et al are circumspect in this regard, recommending that future studies should examine the internal and external carotid arteries separately. We still do not know the extent to which carotid atherosclerosis explains cardiovascular events in RA; thus, it is not possible to issue firm recommendations for management. However, we do know that in the general population, carotid IMT and plaque are strong predictors of $\mathrm{CV}$ events $^{8,11}$. If indeed atherosclerosis is increased in RA as suggested by Kobayashi, et al, it is prudent to manage both $\mathrm{RA}$ and the conventional CV risk factors aggressively ${ }^{18}$. This is especially important in young RA patients, in whom inflammatory factors seem to play a greater role in atherosclerosis ${ }^{14}$. Data on the role of carotid ultrasound in predicting $\mathrm{CV}$ events is needed to further delineate the high-risk groups who would benefit most from atherosclerosis prevention.

INMACULADA DEL RINCON, MD, MS, Division of Rheumatology and Clinical Immunology, Department of Medicine,

University of Texas Health Science Center at San Antonio, 7703 Floyd Curl Drive, San Antonio, Texas 78229, USA

\footnotetext{
Address correspondence to Dr. del Rincon;

E-mail: delrincon@uthscsa.edu

Supported by grants 5RO1 HL085742, 2RO1HD037151, and

UL1RR025767 from the National Institutes of Health.
} 


\section{REFERENCES}

1. Wallberg-Jonsson S, Ohman ML, Dahlqvist SR. Cardiovascular morbidity and mortality in patients with seropositive rheumatoid arthritis in Northern Sweden. J Rheumatol 1997;24:445-51.

2. del Rincon ID, Williams K, Stern MP, Freeman GL, Escalante A. High incidence of cardiovascular events in a rheumatoid arthritis cohort not explained by traditional cardiac risk factors. Arthritis Rheum 2001;44:2737-45.

3. Solomon DH, Karlson EW, Rimm EB, Cannuscio CC, Mandl LA, Manson JE, et al. Cardiovascular morbidity and mortality in women diagnosed with rheumatoid arthritis. Circulation 2003;107:1303-7.

4. Watson DJ, Rhodes T, Guess HA. All-cause mortality and vascular events among patients with rheumatoid arthritis, osteoarthritis, or no arthritis in the UK General Practice Research Database. J Rheumatol 2003;30:1196-202.

5. Turesson C, Jarenros A, Jacobsson L. Increased incidence of cardiovascular disease in patients with rheumatoid arthritis: results from a community based study. Ann Rheum Dis 2004;63:952-5.

6. Maradit-Kremers H, Crowson CS, Nicola PJ, Ballman KV, Roger $\mathrm{VL}$, Jacobsen SJ, et al. Increased unrecognized coronary heart disease and sudden deaths in rheumatoid arthritis: a population-based cohort study. Arthritis Rheum 2005;52:402-11.

7. Kobayashi H, Giles JT, Polak JF, Blumenthal RS, Leffell MS, Szklo $\mathrm{M}$, et al. Increased prevalence of carotid artery atherosclerosis in rheumatoid arthritis is artery-specific. J Rheumatol 2010; 37:730-9.

8. O'Leary DH, Polak JF, Kronmal RA, Manolio TA, Burke GL, Wolfson SK Jr, the Cardiovascular Health Study Collaborative Research Group. Carotid-artery intima and media thickness as a risk factor for myocardial infarction and stroke in older adults. N Engl J Med 1999;340:14-22.

9. Persson J, Formgren J, Israelsson B, Berglund G. Ultrasound-determined intima-media thickness and atherosclerosis: direct and indirect validation. Arterioscler Thromb 1994;14:261-4.
10. Young W, Gofman JW, Tandy R, Malmud N, Waters ESG. The quantification of atherosclerosis, III: the extent of correlation of degrees of atherosclerosis within and between the coronary and cerebral vascular beds. Am J Cardiol 1960;6:300-8.

11. O'Leary DH, Polak JF, Kronmal RA, Kittner SJ, Bond MG, Wolfson SK Jr, et al. Distribution and correlates of sonographically detected carotid artery disease in the Cardiovascular Health Study. Stroke 1992;23:1752-60

12. Wikstrand J. Methodological considerations of ultrasound measurement of carotid artery intima-media thickness and lumen diameter. Clin Physiol Funct Imaging 2007;27:341-5.

13. Roman MJ, Moeller E, Davis A, Paget SA, Crow MK, Lockshin $\mathrm{MD}$, et al. Preclinical carotid atherosclerosis in patients with rheumatoid arthritis. Ann Intern Med 2006;144:249-56. Erratum in: Ann Intern Med 2006;145:868.

14. del Rincon I, Williams K, Stern MP, Freeman GL, O'Leary DH, Escalante A. Association between carotid atherosclerosis and markers of inflammation in rheumatoid arthritis patients and healthy subjects. Arthritis Rheum 2003;48;1833-40.

15. Schott LL, Kao AH, Cunningham A, Wildman RP, Kuller LH, Sutton-Tyrrell K, et al. Do carotid artery diameters manifest early evidence of atherosclerosis in women with rheumatoid arthritis? J Womens Health (Larchmt) 2009;18:21-9.

16. del Rincon I, Freeman GL, Haas RW, O'Leary DH, Escalante A. Relative contribution of cardiovascular risk factors and rheumatoid arthritis clinical manifestations to atherosclerosis. Arthritis Rheum 2005;52:3413-23

17. Aubry MC, Maradit-Kremers H, Reinalda MS, Crowson CS, Edwards WD, Gabriel SE. Differences in atherosclerotic coronary heart disease between subjects with and without rheumatoid arthritis. J Rheumatol 2007;34:937-42.

18. del Rincon I. Atherothrombotic comorbidity in the rheumatic diseases. The evidence becomes clearer. What should clinicians do? Arthritis Rheum 2009;61:1284-6.

J Rheumatol 2010;37:890-3; doi:10.3899/jrheum.100183 